\title{
Alterações na Expressão do Antigeno Nuclear de Proliferaçãa Celular e dos Receptores de Estrogênio e de Progesterona Provocadas pela Quimioterapia Primária no Carcinoma de Mama
}

Assessment of Proliferating Cells of Nuclear Antigen and Estrogen and Progesterone Receptor Expression Changes Determined by Primary Chemotherapy in Breast Cancer

Daniella De Batista Depes, Maria Angela de Souza, Julisa Chamorro Lascasas Ribalta, Maria Tereza de Seixas Alves, Cláudio Kemp, Geraldo Rodrigues de Lima

\section{RESUMO}

Objetivo: averiguar as alterações induzidas pela quimioterapia primária no fenótipo celular. Métodos: avaliamos a expressão do antígeno nuclear de proliferação celular (PCNA) e dos receptores de estrogênio $(R E)$ e de progesterona $(R P)$ em 17 tumores de mama no estádio clínico II, obtidos antes e após a terapia antiblástica, por método imuno-histoquímico. Os valores foram relacionados com o estado menstrual, com a resposta clinica tumoral e com o comprometimento axilar.

Resultados: houve redução significante na porcentagem de células coradas pelo anti-PCNA antes (tempo $A$ ) e após (tempo B) a quimioterapia ( $p=0,041)$. Observamos também resultados significantes ao compararmos os indices médios de PCNA com o grau histológico GII/GIII [tempo $A=63,1$ e tempo $B=38,7(p=0,049)]$ e nos casos em que houve resposta clinica [tempo $A=53,1$ e tempo $B=34,4$ ( $p=0,011)]$. Não observamos relação significante entre os indices de PCNA com o estado menstrual e o axilar. Houve redução significante do RE após a quimioterapia nas pacientes pré-menopausadas [tempo $A=60,3$ e tempo $B=24,1$ $(p=0,027)]$ e naquelas que apresentaram resposta clínica ao tratamento [tempo $A=59,1$ e tempo $B=37,9(p=0,030)]$. Observamos aumento significante do RP após a quimioterapia nas pacientes pós-menopausadas [tempo $A=35,3$ e tempo $B=58,3(p=0,023)]$. Não encontramos relação entre os receptores hormonais e o comprometimento axilar.

Conclusões: a diminuição dos indices de PCNA nos tumores de alto grau histológico, do RE nas pacientes pré-menopausadas e de ambos, PCNA e RE, nos tumores com redução clínica após a quimioterapia nos mostra que ela atuou sobre as células em proliferação e que o PCNA pode ser utilizado como parâmetro de resposta a este tratamento.

PALAVRAS-CHAVE: Quimioterapia. Antigeno nuclear de célula em proliferação. Receptores hormonais. Mama: câncer.

\section{Introdução}

Nas últimas décadas tem-se observado grande revolução no tratamento convencional do carcinoma de mama. Estratégias multidisciplinares fo-

Departamento de Ginecologia, Universidade Federal de São Paulo/Escola Paulista de Medicina (UNIFESP/EPM).

Correspondência:

Daniella De Batista Depes

Rua Canário, 943/164 - Moema

04521-004 - São Paulo - SP - Brasil

Fone: (11) 5561-7831 - Fax: (11) 5088-8153

e-mail: danidepes@yahoo.com.br ram desenvolvidas, baseadas em evidências clínicas e laboratoriais, indicando a natureza sistêmica da doença, já no momento do diagnóstico ${ }^{1}$.

A cirurgia evoluiu para procedimentos menos mutilantes, uma vez que a extensão da retirada do tumor demonstrou exercer pouca influência no prognóstico. Cirurgias conservadoras associadas ao tratamento radioterápico tornaramse eficientes no controle da doença local. A quimioterapia e a hormonioterapia mostraram ser complementos importantes para as manifestações sistêmicas ${ }^{1}$. 
Os resultados obtidos com a utilização da quimioterapia antiblástica primária em mulheres com tumores ressecáveis, com diâmetros iguais ou maiores do que $3 \mathrm{~cm}$, permitiram a preservação da mama em até $94 \%$ dos casos em cinco anos de seguimento. Além dos excelentes resultados cosméticos, esse procedimento possibilitou evidenciar aumento de sobrevida e maior intervalo livre de doença pelo tratamento das micrometástases ${ }^{2}$.

A quimioterapia primária oferecia a vantagem da observação direta da regressão do tumor, possibilitando a escolha correta dos agentes quimioterápicos ou a mudança de esquema, caso não houvesse resposta adequada ${ }^{3}$.

Assim, o emprego mais amplo da quimioterapia primária estimulou a necessidade do desenvolvimento de testes preditivos de respostas, para melhor selecionar as pacientes que se beneficiariam desta modalidade de tratamento ${ }^{4}$.

Como critérios de escolha para o tratamento sistêmico, utilizam-se o tamanho do tumor e o envolvimento linfonodal, nem sempre fatores preditivos acurados de recidiva e de sobrevida. A agressividade do tumor é, muitas vezes, influenciada por fatores de proliferação celular. Assim, a inclusão de marcadores de atividade proliferativa poderia auxiliar na identificação das neoplasias que melhor responderiam à quimioterapia, uma vez que as diferenças de sensibilidade às drogas estão relacionadas aos diferentes estados de proliferação ${ }^{5}$.

Para medir a atividade proliferativa das neoplasias malignas, vários métodos foram idealizados. O antígeno nuclear de proliferação celular, marcador de atividade proliferativa, é facilmente identificável pelo método imuno-histoquímico, sendo seu valor prognóstico demonstrado por vários autores ${ }^{6,7}$.

$O$ receptor de estrogênio ( $R E$ ) tem sido o indicador prognóstico mais intensamente estudado até hoje. Inúmeros autores declararam haver relação positiva entre $\mathrm{RE}$, maior intervalo livre da doença e melhor sobrevida das pacientes ${ }^{8,9}$. Os RE e os receptores de progesterona (RP) são atualmente os fatores preditivos mais utilizados para escolha do tratamento hormonal ${ }^{8,9}$. A seleção das pacientes com RE positivo no tumor primário eleva o nivel de resposta clinica para $60 \%$, ao passo que somente $10 \%$ das pacientes com RE negativo respondem à hormonioterapia. Entretanto ainda permanecem $40 \%$ de pacientes RE positivas que não se beneficiam com o tratamento endócrino ${ }^{8}$.

Uma das maneiras de verificar se o mecanismo de resposta estrogênica está intacto é a determinação do $\mathrm{RP}^{10}$. Relatos recentes têm demonstrado a importância do RP em relação ao prog- nóstico e à resposta clínica ao tratamento endócrino, sendo que essa pode alcançar $75 \%$ quando ambos os receptores (RE e RP) são encontrados nas células tumorais ${ }^{9}$.

O câncer de mama caracteriza-se por constituição celular heterogênea, o que lhe faculta a possibilidade de respostas diferentes à quimioterapia nos diferentes clones celulares dentro do mesmo tumor. Essa heterogeneidade pode ser apontada como uma das razões da resposta negativa à terapia endócrina, em pacientes $\mathrm{RE}$ positivas ${ }^{11}$.

Os métodos imuno-histoquímicos, com os anticorpos monoclonais, vieram facilitar as pesquisas neste campo. Essas técnicas apresentam a vantagem de preservar a morfologia do tecido, permitindo avaliar in situ a proliferação celular, os sítios de expressão do receptor e, por conseguinte, a qualidade da amostra e a heterogeneidade da população de células tumorais ${ }^{7}$.

Considerando a expressão do antígeno nuclear de proliferação celular (PCNA), marcador de atividade proliferativa de alto valor preditivo e, ainda, conhecendo o valor dos receptores hormonais como fatores preditivos da resposta endócrina e procurando elucidar o padrão de modificações induzidas pela quimioterapia antiblástica no câncer de mama, in vivo, sua relação com a resposta ao tratamento e prognóstico, nos propusemos a avaliar as alterações da expressão do PCNA e dos RE e RP antes e após a quimioterapia neoadjuvante.

\section{Pacientes e Métodos}

Neste trabalho prospectivo, aprovado pela Comissão de Ética em Pesquisa da Universidade Federal de São Paulo, selecionaram-se 17 mulheres com carcinoma de mama no estádio clínico II. As pacientes foram tratadas com o esquema ciclofosfamida $-600 \mathrm{mg} / \mathrm{m}^{2}$, metotrexato $-40 \mathrm{mg} /$ $\mathrm{m}^{2}$ e 5 -fluorouracil $-600 \mathrm{mg} / \mathrm{m}^{2}$ por três ciclos com intervalo de 28 dias, em regime neoadjuvante. A idade variou de 36 a 68 anos, com média de 50 anos. Oito das 17 mulheres encontravam-se na pós-menopausa e as demais, na pré-menopausa.

Todos os casos incluídos no estudo tinham tumores unilaterais, pertencentes ao estádio IIA (T2NOM0) ou ao IIB (T2,3N0,1M0). O tamanho do tumor variou de 2,5 a $7,0 \mathrm{~cm}$, com média de 4,4 $\mathrm{cm}$. Os critérios de resposta clínica foram baseados no sistema proposto pela União Internacional de Controle do Câncer (UICC). A conduta cirúrgica definitiva foi a mastectomia radical modificada, com esvaziamento axilar. Observou-se comprometimento linfonodal em 10 casos $(58,82 \%)$. 
Os espécimes histológicos obtidos na biópsia incisional antes do tratamento clínico e os resultantes da mastectomia foram fixados em formol tamponado a $10 \%$, por um período máximo de 24 horas. O estudo anatomopatológico identificou 16 carcinomas ductais e um medular. Quanto ao grau histológico, sete casos foram classificados como bem diferenciados (GI), sete casos com moderada diferenciação (GII), um caso pouco diferenciado (GIII) e um não pôde ser avaliado.

Identificou-se a expressão do PCNA e dos RE e RP pelo método imuno-histoquímico, utilizandose a técnica da estrepto-avidina-biotina-peroxidase para os anticorpos monoclonais anti-PCNA (mouse x PCNA clone PC10 Dako, 1D5 e 1A6). A expressão do PCNA e dos receptores hormonais foi avaliada, utilizando-se o sistema de análise digital de imagem KS 300.

Contaram-se as células de maneira interativa, com aumento de 400 vezes até perfazerem um total de, no mínimo, 1000 células para o PCNA e 100 células para os RE/RP. Somente foram considerados positivos aqueles núcleos com imuno-expressão castanha característica inequívoca, independentemente da intensidade. Os resultados obtidos foram expressos sob a forma de indice, obtido pela relação entre as células coradas e o total de células contadas multiplicado por 100. Utilizou-se o valor de $10 \%$ como limite acima do qual os RE e RP foram considerados positivos.

Referiu-se como tempo A os valores obtidos nas amostras antes da quimioterapia e como tempo B, após o tratamento, ou seja, após os três ciclos de antiblásticos.

\section{Resultados}

Dos 17 casos selecionados, constatou-se resposta clínica à quimioterapia em nove pacientes (53\%). O diâmetro médio dos tumores passou de $4,4 \mathrm{~cm}$ para $2,2 \mathrm{~cm}$ após a quimioterapia, nos casos responsivos.

Observamos ausência de tumor clinicamente palpável em cinco casos, dos quais em dois não foi encontrado tumor residual na peça obtida pela mastectomia. Nos três casos restantes evidenciou-se lesão tumoral apenas na peça cirúrgica.

A porcentagem de células coradas pelo antiPCNA em 15 casos estudados teve média de 54,7\% no tempo A (pré-quimioterapia) e média de $34,4 \%$ no tempo B (após a quimioterapia). O teste $t$ pareado confirmou que o número de células coradas pelo anticorpo PC-10 diminuiu de forma significante $(p=0,041)$ em resposta à quimioterapia (Tabela 1).
Tabela 1 - Correlação da porcentagem de células coradas pelo PCNA, nas 15 pacientes com carcinoma de mama no estádio II no tempo A (antes do tratamento quimioterápico) e no tempo $B$ (após a quimioterapia primária).

\begin{tabular}{ccc}
\hline Caso & Tempo A (\%) & Tempo B (\%) \\
\hline 1 & 31,5 & 41,4 \\
2 & 67,3 & - \\
3 & 80,6 & 2,0 \\
4 & 21,1 & 11,8 \\
5 & 84,5 & - \\
6 & 80,5 & 89,3 \\
7 & 9,0 & 24,3 \\
8 & 74,3 & 34,3 \\
10 & 31,3 & 23,3 \\
11 & 88,6 & 77,6 \\
12 & 19,3 & 1,3 \\
13 & 32,8 & 32,1 \\
14 & 83,5 & 63,4 \\
15 & 39,4 & 23,9 \\
16 & 76,4 & 21,9 \\
Média (erro-padrão) & $54,7(7,3)$ & $34,4(7,5)$ \\
\hline
\end{tabular}

Teste $t$-pareado

PCNA pré $Q T$ T PCNA pós $Q T$

$p=0,041$

Observamos, nos casos em que houve resposta clinica, a ocorrência de diferença significante $(p=0,011)$ entre a porcentagem de expressão do antiPCNA pré e pós-quimioterapia, com média no tempo A de $53,1 \%$ e, no tempo B, de $30,7 \%$ dos casos. Nos casos com ausência de resposta clínica não houve diferença estatisticamente significante (Tabela 2).

Tabela 2 - Correlação da porcentagem de células coradas pelo PCNA nas 15 pacientes com carcinoma de mama no estádio II com a resposta clínica tumoral no tempo A (antes do tratamento quimioterápico) e no tempo B (após a quimioterapia primária).

\begin{tabular}{lcccc}
\hline & \multicolumn{2}{c}{ Resposta negativa } & \multicolumn{2}{c}{ Resposta positiva } \\
& Tempo A & Tempo B & Tempo A & Tempo B \\
\hline & 31,5 & 41,4 & 21,1 & 11,8 \\
& 80,6 & 2,0 & 31,3 & 23,3 \\
& 80,5 & 89,3 & 88,6 & 77,6 \\
& 9,0 & 24,3 & 32,8 & 32,1 \\
& 74,3 & 34,3 & 83,5 & 63,4 \\
& 19,3 & 1,3 & 39,4 & 23,9 \\
\multicolumn{1}{c}{ Média } & 76,4 & 21,9 & 67,3 & - \\
(Erro Padrão) & $(12,0)$ & $(11,3)$ & 84,5 & \\
\hline Teste $t$-pareado & 53,1 & 30,7 & 56,0 & 38,7 \\
Resposta negativa & Resposta Positiva & $(9,8)$ & $(10,6)$ \\
PCNA pré x pós QT & PCNA pré x pós QT & & & \\
p=0,150 & p=0,011 & &
\end{tabular}


Antes da quimioterapia, os valores médios de $\mathrm{RE}$ e de RP foram semelhantes. Após a quimioterapia constatou-se, no grupo de pacientes que apresentou resposta clínica tumoral, redução estatisti- camente significante $(\mathrm{p}=0,030)$ nos níveis de RE. $\mathrm{O}$ mesmo não ocorreu nas pacientes sem resposta clínica $(p=0,54)$ (Tabela 3$)$. Não ocorreram variações nos niveis de RP em nenhum dos grupos.

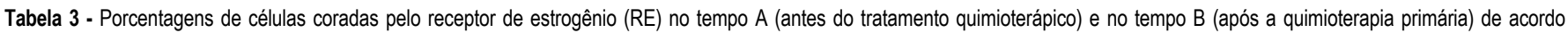
com a resposta tumoral.

RE - Ausência de resposta

Tempo A (\%)

Tempo B (\%)

85

79

72

95

0

99

57

22

$63,6(13,0)$

Presença de resposta

RE Tempo A x Tempo B

$p=0,030$

\section{RE - Presença de resposta}

Tempo A (\%)

Tempo B (\%)

$\begin{array}{cc}32 & - \\ 59 & 0 \\ 0 & - \\ 39 & 0 \\ 94 & 24 \\ 98 & 100 \\ 62 & 0 \\ 96 & 98 \\ 52 & 43\end{array}$

$59,1(11,0)$
Teste $t$-pareado

Ausência de resposta

RE Tempo A x Tempo B

$p=0,540$

Ao compararmos o grau histológico com a variação do anti-PCNA pré e pós-quimioterapia, observamos resultado estatisticamente significante $(p=0,049)$ nos casos do grupo GII/GIII, com média no tempo A de 63,0\% e no tempo B, de 30,0\% (Tabela 4). Nos casos do grupo GI não observamos significância.

Tabela 4 - Correlação da porcentagem de células coradas pelo PCNA nas 15 pacientes com carcinoma de mama no estádio II com o grau histológico no tempo A (antes do tratamento quimioterápico) e no tempo $B$ (após a quimioterapia primária).

\begin{tabular}{|c|c|c|c|c|}
\hline & \multicolumn{2}{|c|}{ Grau histológico I } & \multicolumn{2}{|c|}{ Grau histológico II/III } \\
\hline & Tempo A & Tempo B & Tempo A & TempoB \\
\hline & 31,5 & 41,4 & 80,6 & 2,0 \\
\hline & 80,5 & 89,3 & 21,1 & 11,8 \\
\hline & 9,0 & 24,3 & 74,3 & 34,3 \\
\hline & 31,3 & 23,3 & 88,6 & 77,6 \\
\hline & 19,3 & 1,3 & 76,4 & 21,9 \\
\hline & 83,5 & 63,4 & 67,3 & - \\
\hline & 39,3 & 23,9 & 32,8 & 32,1 \\
\hline Média & 42,1 & 38,2 & 63,0 & 30,0 \\
\hline (erro-padrão) & $(10,9)$ & $(11,2)$ & $(9,7)$ & $(10,7)$ \\
\hline $\begin{array}{l}\text { Teste } t \text {-pareado } \\
\text { Grau I } \\
\text { PCNA pré x pós } Q T \\
p=0,510\end{array}$ & $\begin{array}{l}\text { Grau II/IIII } \\
\text { PCNA pré } \\
p=0,049 \text { ( }\end{array}$ & ós QT & & \\
\hline
\end{tabular}

Em relação ao estado menstrual, a variação do anti-PCNA mostrou não ser significante nas pacientes na pré-menopausa, com média no tempo A de $50,4 \%$ e no tempo B, de $25,6 \%$. Observouse, entretanto, tendência $(p=0,052)$ nos casos na pós-menopausa, com média no tempo A de $59,6 \%$ e no tempo B, de $44,6 \%$.

Observamos que as médias dos valores percentuais de RE e de RP nas pacientes pré e pósmenopáusicas eram semelhantes antes da quimioterapia. O valor médio do RE após a quimioterapia primária mostrou diminuição estatisticamente significante nas pacientes pré-menopáusicas $(\mathrm{p}=0,027)$, não se alterando naquelas na pós-menopausa $(\mathrm{p}=0,920)$ (Tabela 5$)$. O valor percentual médio do RP nas pacientes na pós-menopausa após a quimioterapia primária mostrou aumento estatisticamente significante $(p=0,023)$, não se alterando naquelas na pré-menopausa $(\mathrm{p}=0,98)$.

Quanto ao estado dos linfonodos axilares e a variação do anti-PCNA, houve tendência estatística $(p=0,064)$ nos casos com axila negativa, com média de valor no tempo A de $58,9 \%$ e no tempo B, de $20,2 \%$. Nos casos com axila positiva, não houve diferença de valor, sendo a média no tempo A de $51,9 \%$ e no tempo B, de $40,6 \%$. As médias dos valores de RE e RP pré-quimioterapia mostraram-se semelhantes, estando ou não a axila comprometida. Após a quimioterapia, não ocorreu alteração nos níveis médios de ambos os receptores. 
Tabela 5 - Porcentagens de células coradas pelo receptor de estrogênio (RE) nas 17 pacientes com carcinoma de mama no estádio clínico II no tempo A (antes do tratamento quimioterápico) e no tempo B (após a quimioterapia primária) de acordo com o estado menstrual.

\begin{tabular}{lcccc}
\hline & \multicolumn{2}{c}{ RE - Pré-menopausa } & \multicolumn{2}{c}{ RE - Pós-menopaua } \\
& Tempo A (\%) & Tempo B (\%) & Tempo A (\%) & Tempo B (\%) \\
& 85 & 0 & 59 & 0 \\
& 32 & - & 0 & 75 \\
& 79 & 0 & 72 & 100 \\
& 95 & 93 & 98 & 74 \\
& 0 & 0 & 99 & 98 \\
& 39 & 0 & 96 & 43 \\
Média (Erro-padrão) & 94 & 24 & 52 & 97 \\
\hline - desaparecimento do tumor & 62 & 0 & 22 & $69,6(14,0)$ \\
Pré-menopausa & 57 & 76 & &
\end{tabular}

\section{Discussão}

A influência dos quimioterápicos no fenótipo dos carcinomas de mama tem sido alvo de estudos por vários autores há cerca de três décadas. A equação de Gompertz mostra que à medida que o tumor cresce aumenta o tempo de duplicação do seu volume, isto é, haveria lentidão no crescimento exponencial. Como os quimioterápicos interagem com as células mitoticamente ativas, há menor potencial de morte celular nas massas maiores que, por sua vez, apresentam menores frações de crescimento. Portanto, há um periodo inicial, no crescimento do tumor, durante o qual a cura é possivel com quimioterápicos. Esses serão ineficazes nos estádios mais tardios.

Vários são os estudos mostrando a influência da cinética celular na resposta à quimioterapia em casos de câncer mamário avançado, sempre utilizando diversos esquemas terapêuticos, evidenciando que a avaliação da atividade proliferativa poderia ser fator preditivo de resposta ${ }^{4}$.

Como os vários estudos que avaliaram a resposta tumoral à quimioterapia foram feitos em casos avançados e com diferentes esquemas de quimioterápicos, somamos as considerações acima citadas e concentramos nossos objetivos em estudar tumores no estádio II, com esquema único, para melhor avaliar a resposta à quimioterapia.

Uma vez que a atuação dos quimioterápicos está estritamente ligada ao ciclo celular, os estudos dos marcadores de proliferação celular poderiam mostrar alterações sob a ação da quimioterapia.
Utilizamos, como marcador de atividade proliferativa, a expressão do PCNA, proteína da polimerase$\mathrm{d}$ existente principalmente em células onde ocorre a replicação ou síntese do DNA.

A resposta clínica à quimioterapia nos tumores mamários varia de 50 a $88 \%$ dos casos ${ }^{2}$. Esta variação está relacionada ao tamanho do tumor, sendo os melhores índices encontrados nos tumores $\mathrm{T}_{2}$ e os piores, nos $\mathrm{T}_{3} \mathrm{O}$ valor encontrado em nosso estudo, referente à resposta clínica posterior à quimioterapia, foi de 53\% e está de acordo com os dados relatados na literatura ${ }^{2}$.

O achado de $11 \%$ (dois casos) com resposta completa, inclusive na análise da peça cirúrgica, está de acordo com a literatura, que mostra porcentagem de 10 a $20 \%{ }^{2}$.

Outro fator a considerar quanto à importância da resposta clínica é a possibilidade de oferecer, ao médico e à paciente, a observação da regressão do tumor, permitindo a escolha correta do tratamento sistêmico e do tipo de cirurgia. Segundo Bonadonna et al. $^{2}$, a transitória inconveniência produzida pela quimioterapia foi preferida por suas pacientes à mutilação permanente. Em nosso estudo, nove pacientes responderam com redução do tumor para medidas que permitiriam a cirurgia conservadora. Para demonstrar parâmetros de segurança na indicação desta modalidade de tratamento passamos, agora, a discutir nossos resultados em relação ao marcador de atividade celular tumoral.

Os valores percentuais médios de antiPCNA de $54,7 \%$ no tempo A e de $34,4 \%$ no tempo B observados em nosso material estão dentro do intervalo de variação encontrado na literatura, ou 
seja, de 10 a $60 \%$ de células coradas ${ }^{7}$. Explicam esta variação a heterogeneidade intratumoral, diferentes técnicas e tempo de fixação, métodos de contagem ou ainda a variabilidade do observador ao interpretar as amostras.

Analisando os resultados deste estudo, observou-se que a quimioterapia reduziu, de maneira estatisticamente significante, a atividade proliferativa dos cânceres de mama no estádio II, com diminuição dos índices de anti-PCNA após a terapia citotóxica. O principal modo de ação das drogas citotóxicas é a morte das células em divisão. De acordo com conceitos de cinética celular de Skipper et al. ${ }^{12}$, frações constantes de uma população celular tornam-se inviáveis diante de determinada concentração de antiblásticos e essas frações são tanto maiores quanto mais elevados os indices proliferativos teciduais. Assim, é lícito esperar a redução do índice de proliferação pela efetiva quimioterapia primária.

Nos casos em que se verificou resposta, isto é, nos casos em que houve redução do tumor maior ou igual a $50 \%$, a variação do PCNA foi estatisticamente significante e todos os casos apresentaram queda dos indices.

$\mathrm{Na}$ analise dos nossos resultados, encontramos significativa redução nos niveis de RE nas pacientes que tiveram resposta à quimioterapia. Resultados semelhantes foram também relatados por Frassoldati et al. ${ }^{13}$. O emprego de drogas citotóxicas pode eliminar células que contenham receptores hormonais ou alterar a sua concentração (Clarke et al., 1997) ${ }^{14}$.

Não encontramos associação entre positividade para os receptores e maior resposta clínica à quimioterapia, porém, é fato conhecido que os RE e RP têm valor para predizer a resposta ao tratamento hormonal, mas não à quimioterapia antiblástica. Nosso índice de resposta clínica tumoral, de maneira semelhante à encontrada por Bonadonna et $a .^{2}$, não teve relação com a idade e com o estado menstrual da paciente.

Ao avaliar o grau histológico, observamos que não houve diferença de graduação entre os espécimes estudados antes e após a quimioterapia. Outros autores confirmaram estes resultados, concluindo que o grau histológico do carcinoma ductal invasivo residual após a quimioterapia parece refletir a graduação do espécime obtido na biópsia incisional ${ }^{15}$.

Ao correlacionar o grau histológico com a porcentagem de células coradas pelo PCNA, antes e após a quimioterapia, notamos diminuição significante dos índices nos casos GII/GIII . Este achado está de acordo com a afirmação feita por Mortimer e Taylor ${ }^{16}$ sobre a diminuição da população de células ser esperada nos tumores de maior grau histológico, refletindo a resposta à quimioterapia nos tumores mais agressivos, isto é, com maior taxa de proliferação.

A presença do receptor, segundo Memon e Given ${ }^{17}$, independe do tamanho do tumor e do comprometimento axilar, fato também desvelado em nosso estudo.

Em relação ao estado menstrual, encontramos importante redução dos valores de RE nas pacientes na pré-menopausa. O carcinoma mamário em mulheres jovens mostra altos índices de proliferação (tumores indiferenciados). A falência ovariana precoce nas pacientes jovens e, a conseqüente redução estrogênica responsabilizarse-iam por alguns dos efeitos do tratamento, como a redução dos níveis de $\mathrm{RE}$. Quando avaliamos o comportamento dos RP, observamos que houve aumento estatisticamente significante após a quimioterapia em pacientes na pós-menopausa. Nessas pacientes os niveis de RE pós-quimioterapia mantiveram-se altos, o que justifica por si o aumento proporcional dos valores de RP.

Katzenellenbogen e Norman ${ }^{18}$ demonstraram que o RP, freqüentemente considerado sob exclusiva regulação extrínseca é regulado também por outros hormônios, como a insulina e o IGF-1 (fator de crescimento tipo insulina-1), dentre outros. De fato, demonstrou-se que a concentração dos receptores de IGF-1 em células de câncer mamário, correlaciona-se linearmente com os níveis de RP e RE, sugerindo haver ação endócrina, parácrina e autócrina na estimulação do crescimento tumoral e nos índices de RP.

Não observamos, ainda, correlação entre os niveis de RE antes do tratamento e o estado menstrual. Este achado é justificado pelo fato de que nossa metodologia detecta o RE aditivamente, independentemente de estar ligado ao estradiol endógeno, de modo diferente do ensaio bioquímico, que detecta RE livres, presentes em maior número nos tumores de pacientes na pós-menopausa ${ }^{19}$.

Os estudos de cinética celular realizados em carcinomas mamários são concordes com o observado para outros tipos de tumores humanos. A atividade proliferativa do tumor primário é uma variável, por vezes independente das características anatomopatológicas e um importante indicador prognóstico ${ }^{20}$.

Analisando, em conjunto, a diminuição significante do valor médio de PCNA após a quimioterapia, nos casos com grau histológico GII/GIII e naqueles com resposta clínica, conclui-se que a quimioterapia agiu sobre células em proliferação e que o índice de PCNA pode ser utilizado como parâmetro de resposta, principalmente nos tumores mais agressivos ${ }^{21,22}$. 
O câncer de mama é heterogêneo. As pacientes apresentam diferentes estádios de desenvolvimento da doença, além da alta variabilidade inerente ao tumor, mostrando distintas taxas de crescimento tumoral, padrão de metástase e outras características biológicas. Assim, o resultado de determinado tratamento varia de uma paciente para outra ${ }^{5}$.

Não se sabe se as alterações observadas após a quimioterapia primária são apenas alterações do fenótipo celular ou se traduzem a expressão de novos clones neoplásicos. Porém, se as células do câncer alteram sua aparência durante a QT primária, devemos estar atentos para a necessidade de adaptarmos a nossa estratégia de tratamento a fim de melhorarmos os resultados.

\section{ABSTRACT}

Purpose: to evaluate the changes in the cell phenotype determined by primary chemotherapy.

Methods: we evaluated the expression of proliferating cells of nuclear antigen (PCNA) and the estrogen (RE) and progesterone (RP) receptors in 17 stage II breast cancer patients before and after chemotherapy by immunohistochemistry. The values were compared with menopausal status, tumoral clinical response and with axillary lymph node status.

Results: there was a significant decrease in the average index of anti-PCNA-stained cells before (time A) and after (time B) chemotherapy $(p=0.041)$. Responder patients displayed a significant decrease in PCNA levels [time $A=53.1$ and time $B=30.7(p=0.011)]$. A similar trend was observed in patients with histologic grade GII/GIII [time $A=63.1$ and time $B=38.7$ $(p=0.049)]$. There was no significant difference in PCNA expression regarding menopause status and axillary lymph node involvement. There was a significant decrease in $R E$ after chemotherapy in the premenopausal patients [time $A=60.3$ and time $B=24.1(p=0.027)]$ and in those who showed a therapeutic response [time $A=59.1$ and time $B=37.9$ $(p=0.030)]$. We observed a significant increase in RP after chemotherapy in the postmenopausal patients [time $A=35.3$ and time $B=58.3(p=0.023)]$. There was no relationship between hormone receptors and axillary lymph nodes.

Conclusions: the decrease in PCNA levels in patients with high histologic grade, in RE in premenopausal patients, and both, PCNA and RE, in the tumors with clinical response after chemotherapy shows that the drugs acted on proliferating cells, and therefore PCNA can be used as a parameter of treatment response.

KEYWORDS: Breast cancer. Chemotherapy. Proliferating cells of nuclear antigen. Hormone receptors.

\section{Referências}

1. Bonadonna G. Karnofsky memorial lecture. Conceptual and practical advances in the management of breast cancer. J Clin Oncol 1989; 7:1380-97.

2. Bonadonna G, Veronesi U, Brambilla C, et al. Primary chemotherapy to avoid mastectomy in tumors with diameters of three centimeters or more. J Natl Cancer Inst 1990; 82:1539-45.

3. Scholl S M, Fourquet A, Asselain B, et al. Neoadjuvant versus adjuvant chemotherapy in premenopausal patients with tumours considered too large for breast conserving surgery: preliminary results of a randomised trial: S6. Eur J Cancer 1994; 30A:645-52.

4. Chevillard S, Pouillart P, Beldjord C, et al. Sequential assessment of multidrug resistance phenotype and measurement of S-phase fraction as predictive markers of breast cancer response to neoadjuvant chemoterapy. Cancer 1996; 77:292-300.

5. Henderson IC, Patek AJ. The relationship between prognostic and predictive factors in the management of breast cancer. Breast Cancer Res Treat 1998; 52:261-88.

6. Kemp C, Alberti VN, Lima GR, Carvalho FM. How should PCNA be assessed? Total of stained cells or only the most intensely stained ones? São Paulo Med J 1998; 116:1667-74.

7. Haerslev T, Jacobsen GK, Zedeler K. Correlation of growth fraction by Ki-67 and proliferating cell nuclear antigen (PCNA) immunohistochemistry with histopathological parameters and prognosis in primary breast carcinomas. Breast Cancer Res Treat 1996; 37:101-13.

8. Allegra JC, Lippman ME, Thompson EB, et al. Estrogen receptor status: an important variable in predicting response to endocrine therapy in metastatic breast cancer. Eur J Cancer 1980; 16:323-31.

9. Chevallier B, Heintzmann F, Mosseri V, et al. Prognostic value of estrogen and progesterone receptors in operable breast cancer. Results of a univariate and multivariate analysis. Cancer 1988; 62:2517-24.

10. Horowitz KB. Progesterone receptors and hormone dependent breast cancer [thesis]. Dallas: Univ. of Texas; 1975.

11.Poulsen HS, Jensen J, Hermansen C. Human breast cancer: heterogeneity of estrogen binding sites. Cancer 1981; 48:1791-3.

12.Skipper HE, Schabel FM, Wilcox WS. Experimental evaluation of potential anti-cancer agents. XIII. On criteria and kinetics associated with curability of experimental leukaemia. Cancer Chemother Rep 1964; (35):3-111.

13.Frassoldati A, Adami F, Banzi C, Criscuolo M, Piccinini L, Silingardi V. Changes of biological features in breast cancer cells determined by primary chemotherapy. Breast Cancer Res Treat 1997; 44:185-92. 
14. Clarke RB, Howell A, Potten CS, Anderson E. Dissociation between steroid receptor expression and cell proliferation in the human breast. Cancer Res 1997; 57:4987-91.

15. Frierson HF Jr, Fechner RE. Histologic grade of locally advanced infiltrating ductal carcinoma after treatment with induction chemotherapy. Am J Clin Pathol 1994; 102:154-7.

16. Mortimer JE, Taylor ME. Can chemotherapy after the histologic features of breast cancer? Am J Clin Pathol 1994; 102:147-9.

17. Memon I, Given F. Correlation of oestrogen receptors with prognostic indices in primary breast carcinoma. Ir Med J 1986; 79:90-3.

18.Katzenellenbogen BS, Norman MJ. Multihormonal regulation of the progesterone receptor in MCF-7 human breast cancer cells: interrelationships among insulin/insulin-like growth factor-I, serum and estrogen. Endocrinology 1990;126:891-8.
19. Chua DY, Pang MW, Rauff A, Aw SE, Chan SH. Correlation of steroid receptors with histologic differentiation in mammary carcinoma: a Singapore experience. Cancer 1985; 56:2228-34.

20.Silvestrine R. Contribuição dos estudos de cinética celular no prognóstico do carcinoma mamário. In: Veronesi U, editor. Câncer de Mama. $1^{\text {a }}$ ed. São Paulo: Ícone; 1993. p.53-62.

21.Montesco MC, Zavagno G, Meggiolaro F, et al. Vimentin and proliferating cell nuclear antigen (PCNA) expression in node-negative breast carcinomas and their correlations with pathologic variables and prognosis. Breast 1995; 4:175-8.

22.Rasbridge SA, Gillett CE, Seymour AM, et al. The effects of chemotherapy on morphology, cellular proliferation, apoptosis and oncoprotein expression in primary breast carcinoma. Br J Cancer 1994; 70:335-41.

Recebido em: 28/8/2003 Aceito com modificações em: 15/9/2003

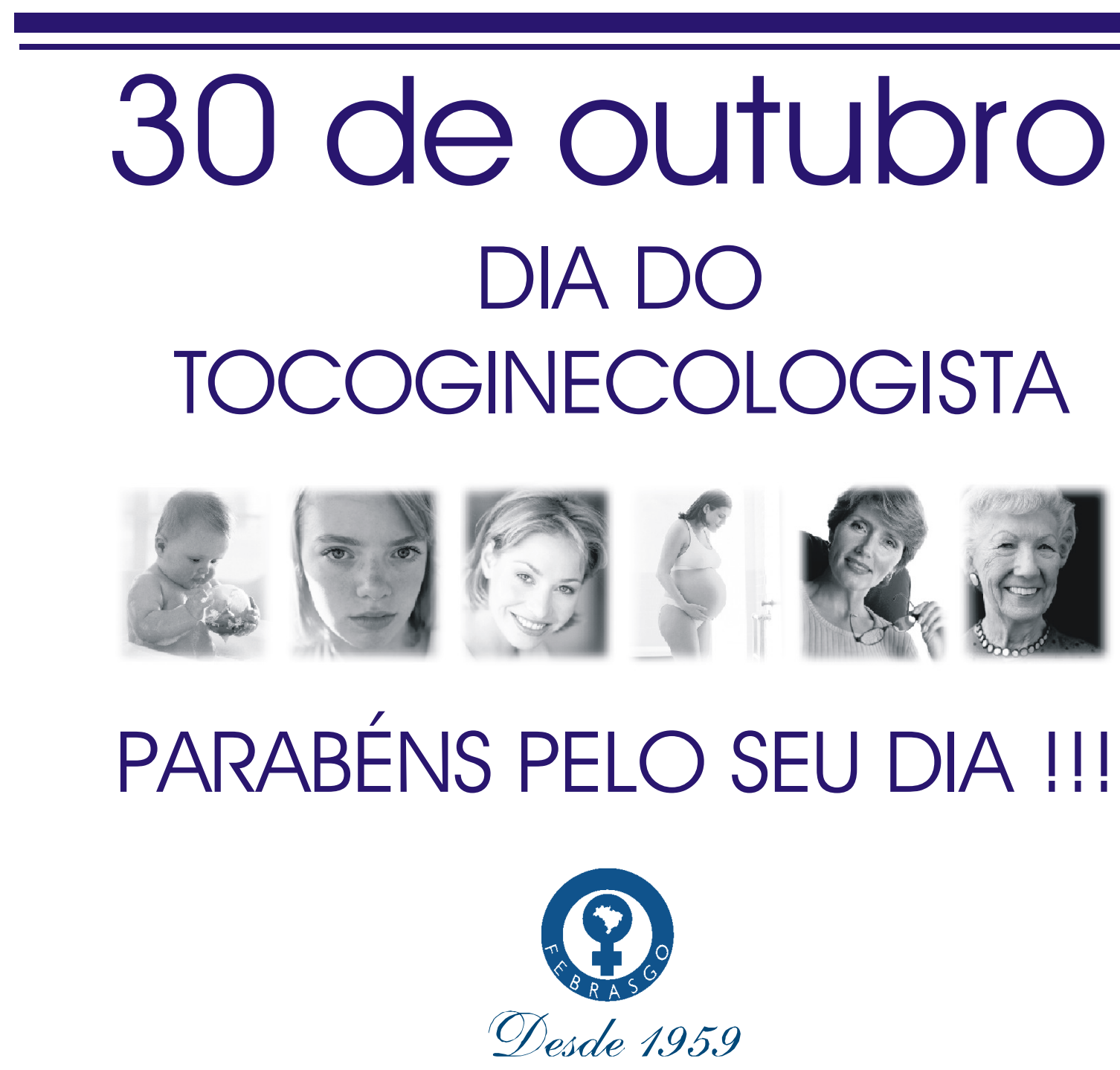

